\title{
Human urine-derived renal epithelial cells provide insights into kidney-specific alternate splicing variants
}

\author{
Elisa Molinari ${ }^{1} \cdot$ Eva Decker $^{2} \cdot$ Holly Mabillard $^{3} \cdot$ James Tellez $^{4} \cdot$ Shalabh Srivastava $^{1} \cdot$ Shreya Raman $^{5}$. \\ Katrina Wood ${ }^{5}$ - Caroline Kempf ${ }^{6}$. Sumaya Alkanderi ${ }^{1}$ - Simon A. Ramsbottom ${ }^{1}$ - Colin G. Miles ${ }^{1}$. \\ Colin A. Johnson $\mathbb{D}^{7} \cdot$ Friedhelm Hildebrandt $\mathbb{C}^{8} \cdot$ Carsten Bergmann $^{2,9} \cdot$ John A. Sayer $\mathbb{D}^{1,3}$
}

Received: 23 March 2018 / Revised: 13 June 2018 / Accepted: 19 June 2018 / Published online: 12 July 2018

(c) The Author(s) 2018. This article is published with open access

\begin{abstract}
The majority of multi-exon genes undergo alternative splicing to produce different mRNA transcripts and this may occur in a tissue-specific manner. Assessment of mRNA transcripts isolated from blood samples may sometimes be unhelpful in determining the affect on function of putative splice-site variants affecting kidney-specific mRNA transcripts. Here we present data demonstrating the power of using human urine-derived renal epithelial cells (hUREC) as a source of kidney RNA. We report clinical and molecular genetic data from three affected cases from two families all with end-stage renal disease by 15 years of age. In both families, heterozygous variants which are predicted to effect function in NPHP3 were found on one allele, in combination with a synonymous SNV (c.2154C >T; p.Phe718=), 18 base pairs from the exon-intron boundary within exon 15 of NPHP3. The only mRNA transcript amplified from wild-type whole blood showed complete splicing out of exon 15. Urine samples obtained from control subjects and the father of family 2, who carried the synonymous SNV variant, were therefore used to culture hUREC and allowed us to obtain kidney-specific mRNA. Control kidney mRNA showed retention of exon 15, while the mRNA from the patient's father confirmed evidence of a heterozygous alternate splicing of exon 15 of NPHP3. Analysis of RNA derived from hUREC allows for a comparison of kidney-specific and whole-blood RNA transcripts and for assessment of the effect on function of putative splice variants leading to end-stage kidney disease.
\end{abstract}

\section{Introduction}

Next-generation sequencing techniques, including wholeexome sequencing (WES), has seen a huge surge in gene discovery, exemplified by gene discovery in various forms of inherited renal diseases. Indeed, ciliopathy exome

Electronic supplementary material The online version of this article (https://doi.org/10.1038/s41431-018-0212-5) contains supplementary material, which is available to authorized users.

John A. Sayer

John.sayer@ncl.ac.uk

1 Institute of Genetic Medicine, Newcastle University, Central Parkway, Newcastle NE1 3BZ, UK

2 Center for Human Genetics, Bioscientia, Ingelheim, Germany

3 Renal Services, Newcastle upon Tyne Hospitals NHS Foundation Trust, Newcastle NE7 7DN, UK

4 Northern Genetic Service, Newcastle upon Tyne NHS Foundation Trust, Newcastle NE1 3BZ, UK capture and WES approaches have been successful in identifying numerous new genetic forms of nephronophthisis, an autosomal recessive renal ciliopathy [1-3]. Similarly, isolation of whole-blood RNA followed by RTPCR strategies for identifying variants in sets of large genes underlying certain phenotypes has been successful [4]. Routine genetic diagnostics typically verify splicesite variants identified by genetic and genomic approaches using whole-blood or fibroblast RNA to assess for aberrant splicing. This has the advantage of assessing RNA

5 Royal Victoria Infirmary, Newcastle upon Tyne Hospitals NHS Foundation Trust, Newcastle, UK

6 Department of Pediatric Nephrology, Charité University Hospital, Berlin, Germany

7 Faculty of Medicine \& Health, Leeds Institute of Biomedical and Clinical Sciences, University of Leeds, Leeds LS9 7TF, UK

8 Department of Medicine, Boston Children's Hospital, Harvard Medical School, Boston, MA, USA

9 Department of Medicine, University Hospital Freiburg, Freiburg, Germany 
directly from the patient (rather than using in silico tools) but the disadvantage is that RNA from affected tissues is not usually available and the gene of interest may not be expressed in the available tissue. For example, assessment of mRNA transcripts isolated from blood samples may be unhelpful in determining the functional effect of putative splice-site variants affecting kidney-specific mRNA transcripts. In the modern post-genome sequencing era, interpretation and functional validation of genomic variants has become vital in the understanding of disease. Genetic variants may have consequences on RNA processing, including exon skipping, intron inclusion, and cryptic splicing. The majority of multi-exon genes undergo alternative splicing to produce different mRNA transcripts and this occurs in a tissue-specific manner [5]. Efforts to understand tissue-specific RNA transcripts have been made [6], which will hopefully increase our understanding of genotype/phenotype correlation in inherited diseases and allow the verification of the consequences of genomic variants in appropriate tissues.

Nephronophthisis is a chronic interstitial nephropathy and an autosomal recessive form of childhood kidney failure. It has numerous genetic causes, although the underlying molecular genetic change remains elusive in around $50 \%$ of cases, due to either unknown genetic causes or hidden variants in known genes. A molecular genetic diagnosis is important to understand genotype/phenotype correlations, prevent the need for a renal biopsy, and to perform screening of at risk individuals.

Here we present data demonstrating the power of using human urine-derived renal epithelial cells (hUREC) as a liquid biopsy of the kidney in order to isolate tissue-specific RNA, to demonstrate the functional effect of a genomic DNA variant in NPHP3 found in families with previously undiagnosed end-stage renal disease (ESRD).

\section{Methods}

All patients consented to this study. Ethical approval was obtained from the UK National Research Ethics Service Committee Northern and Yorkshire (09/H0903/36). Following informed and written consent, blood samples were obtained from affected patients and their relatives and healthy gender and age-matched controls. WES was performed as previously described [7] and targeted sequence analysis was performed using a customized sequence capture library [8]. hURECs were isolated from urine collected from the father of family 2 and healthy age-matched donors and cultured as previously described $[9,10]$. Gene variants have been submitted to www.LOVD.n1/NPHP3 (patient IDs 00164069, 00164068, and 00164805). Full details of methodology are provided in Supplementary Data.

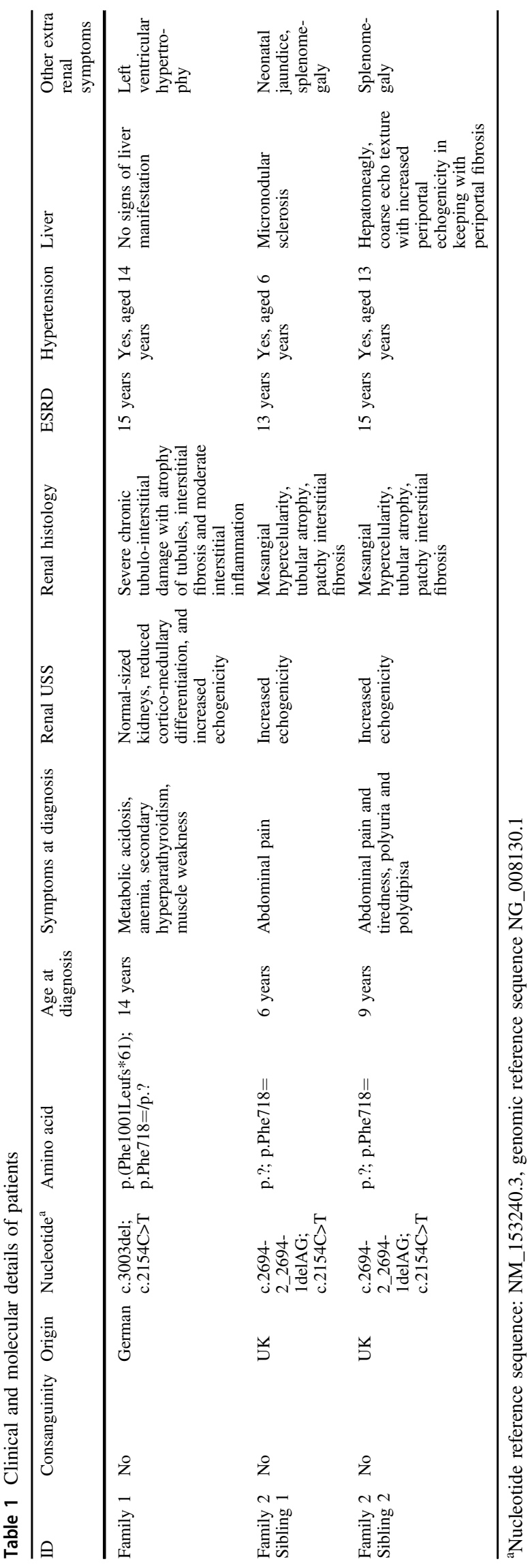


Table 2 In silico analysis of NPHP3 variants

\begin{tabular}{lllll}
\hline NPHP3 variant $^{\mathrm{a}}$ & MutationTaster & $\begin{array}{l}\text { Human Splicing } \\
\text { Finder }\end{array}$ & ExAC & gnomAD \\
\hline c.3003del; p.(Phe1001Leufs*61) & Disease causing, likely NMD & n/a & Not in ExAC & Not in gnomAD \\
c.2694-2_2694-1delAG; p.?; & Disease causing_splice acceptor variant & Acceptor site loss & 43 alleles in & 77 alleles in \\
& (HGMD CD082161; rs751527253) & 121,030 alleles & 277,098 alleles \\
c.2154C>T; p.Phe718= & Disease causing (rs558637226) & "Possible branch & 1 allele in & 3 alleles in \\
& & point motif broken & 121,283 alleles & 277,341 alleles \\
\hline
\end{tabular}

NMD nonsense-mediated decay

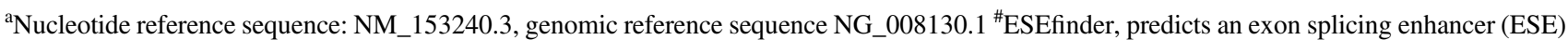
site is abolished thus inhibiting the binding of splicing enhancer proteins leading to aberrant splicing

\section{Results}

\section{Clinical features and genomic investigations of two families with unexplained ESRD}

In the first family, a 14-year-old presented chronic kidney disease stage 4 which progressed to unexplained ESRD by 15 years of age (Table 1). The parents were nonconsanguineous and white European. There was no family history of renal disease. Renal ultrasound showed increased echogenicity and loss of cortico-medullary differentiation and a renal biopsy showed chronic interstitial fibrosis and tubular atrophy suggestive of nephronophthisis (Table 1).

In the second family, two siblings presented with congenital hepatic fibrosis and unexplained ESRD. The first sibling received a combined kidney and liver transplant aged 15 years (Table 1). A renal biopsy had demonstrated glomerular sclerosis and interstitial fibrosis with features suggestive of nephronophthisis (Supplementary Fig. S1). The second sibling reached ESRD at the age of 13 years and had received a renal transplant followed by a liver transplant 1 year later. The family was white British and nonconsanguineous and had an unaffected male sibling (Table 1).

In Family 1, targeted exon capture and sequencing of a ciliopathy panel of 121 genes, as previously reported $[8,11]$, identified a variant predicted to affect function in NPHP3 (NM_153240.3, c.3003del; p.(Phe1001Leufs*61)) in combination with a synonymous SNV (c.2154C >T; p. Phe718=). The variants were present in compound heterozygous state with the frameshift variant inherited maternally and the synonymous change inherited paternally (Table 2, Fig. 1).

In family 2, following exclusion of $P K H D I$ variants by direct sequencing, WES revealed a single heterozygous variant predicted to affect function (c.2694-2_2694-1delAG) in NPHP3, segregating from the mother, combined with the above-mentioned synonymous SNV in NPHP3 (c.2154C $>\mathrm{T}$; p.Phe718=), segregating from the father (Table 2). The functional effect of the c.2694-2_2694-1delAG variant in leading to abnormal splicing was confirmed using RT-PCR from whole-blood RNA in the affected siblings of family 2 (Supplementary Fig. S2). In both families, the rare synonymous SNV in NPHP3 (Table 2) was initially assumed to be benign and has been reported within ClinVar as "likely benign" (SCV000316262.1). However, given this was identified in three individuals from two different families with overlapping clinical phenotypes and the fact that no other known ciliopathy gene variants affecting function had been identified using NGS approaches in these families, additional investigations were undertaken.

The NPHP3 c. $2154 \mathrm{C}>\mathrm{T}$ variant is near an exon-intron boundary (exon 15-intron 15), and therefore may have been implicated in abnormal splicing of exon 15 of NPHP3. In silico tools, including Human Splicing Finder suggested a functional effect, with probable disruption of exonic splicing motifs (Table 2).

mRNA isolated from whole blood from the affected patients from family 2 and unaffected healthy controls all showed complete splicing out of exon 15 of NPHP3, which was confirmed by Sanger sequencing, suggesting RNA splicing of this exon was tissue specific (Fig. 1). Both affected patients in family 2 had received a renal transplant, therefore RNA isolated from their urine would represent the donor. Instead, urine samples obtained from the father in family 2 and healthy control subjects were used to culture hUREC, allowing us to obtain kidney-specific mRNA. Using these tissue-derived mRNA samples, RT-PCR across exon 15 of $N P H P 3$ in control kidney mRNA showed retention of exon 15, confirmed by Sanger sequencing, while the RNA from the father from family 2 showed heterozygous alternative splicing in NPHP3, confirming a kidney-specific loss of function of the c.2154C $>\mathrm{T}$ allele.

\section{Discussion}

Variants that affect function in NPHP3 can lead to a variety of clinical presentations, as emphasized by the two families reported here. Although reported initially as a cause of adolescent NPHP [12], variants that affect function in 
A Family 1 c.2154C $>T ; p . F 718 F$ c.3003del; p.F1001Lfs*61
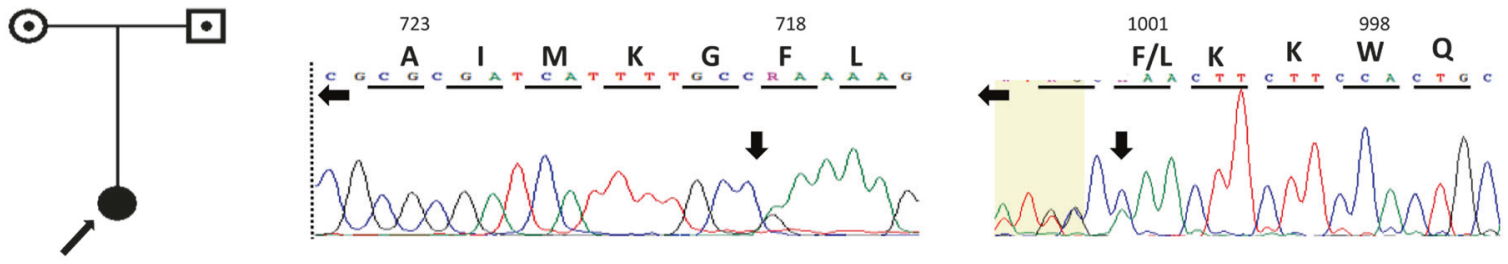

B
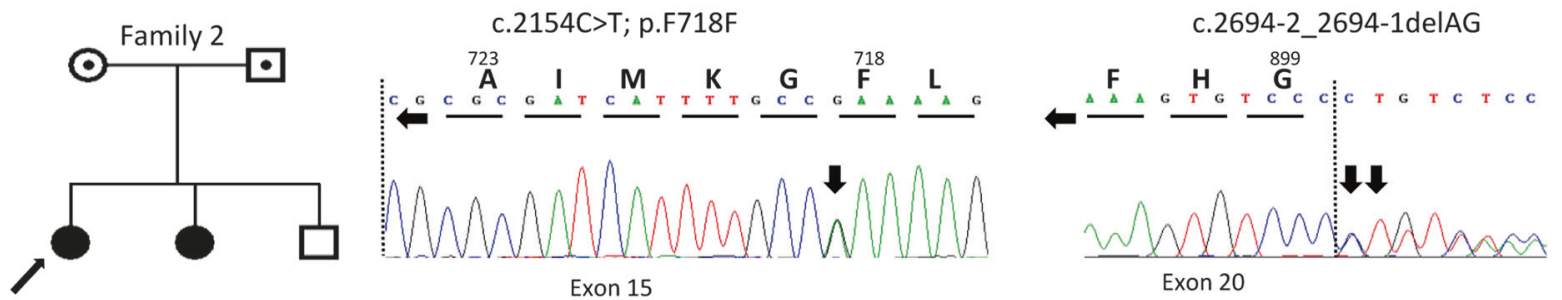

C

D
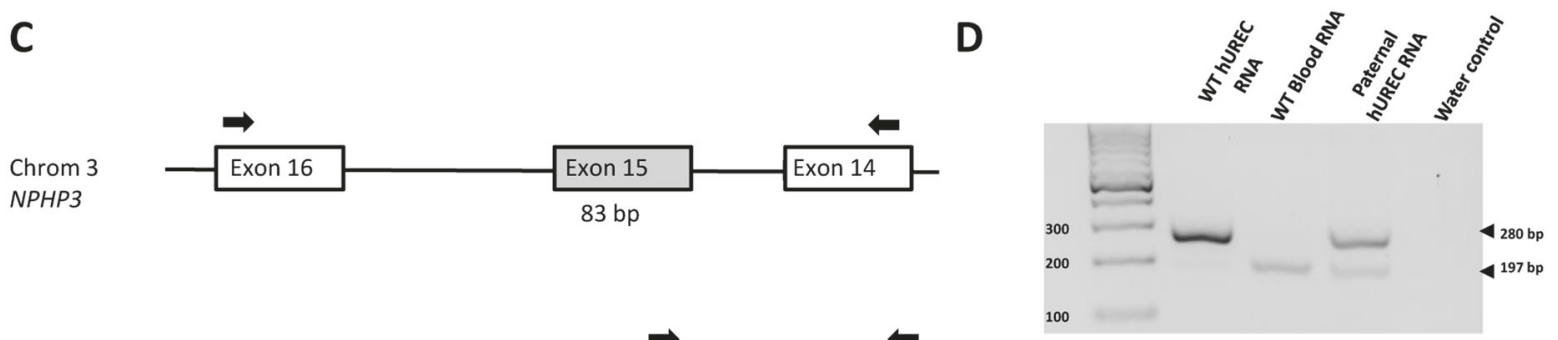

E RT-PCR whole blood mRNA $197 \mathrm{bp}$ product

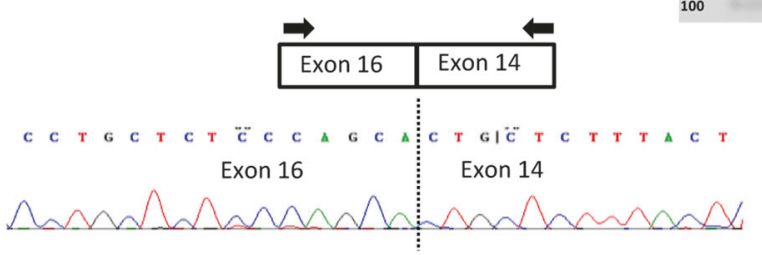

F RT-PCR wild type kidney cell mRNA 280 bp product
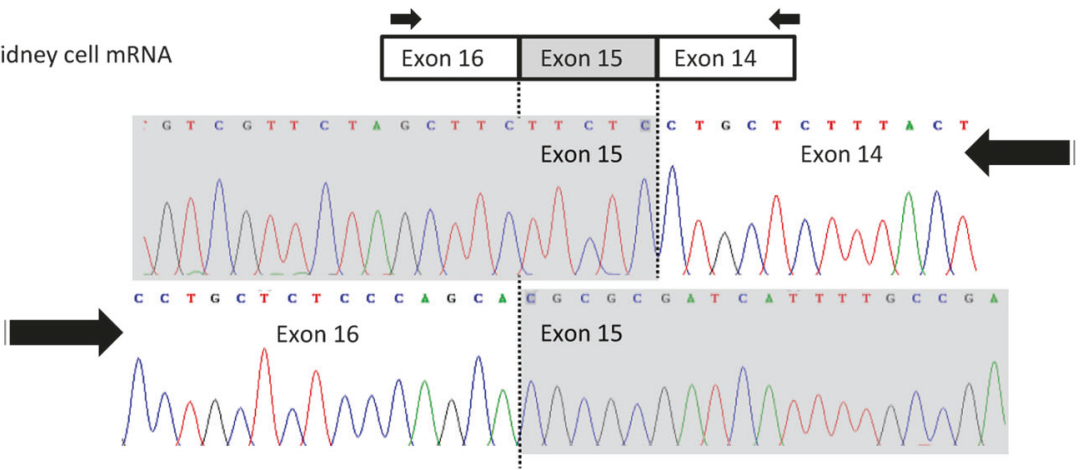

Fig. 1 Molecular genetic investigation and tissue-specific RT-PCR reveals a functional effect of a synonymous NPHP 3 allele. a, b Pedigree diagrams (males squares, females circles, probands arrowed) and sequence chromatograms showing biallelic variants in NPHP3 in affected patient in a Family 1 and b Family 2. c Genomic map and RTPCR strategy to detect abnormal splicing of exon 15 of NPHP3, with primers arrowed. Exons are numbered as in Olbrich et al. [12]. d RT-

NPHP3 are a prominent cause of infantile NPHP and may present antenatally with enlarged cystic kidneys [13] and sometimes cause the perinatally lethal Meckel Syndrome [14]. The wide phenotypic variability, especially in extra-
PCR using RNA isolated from wild-type (WT) hUREC, WT Whole blood and family 2 paternal hUREC. Note paternal RNA is compound heterozygous for alternate splicing of exon 15 of NPHP3 (NM_153240.3: c.2154C $>$ T; r.2154c $>$ u). e Sanger sequencing confirms alternate splicing of NPHP3 exon 15 in wild-type whole-blood RNA. f Sanger sequencing confirms inclusion of exon 15 in RNA derived from wild-type hUREC

renal manifestations may sometimes be explained by the predicted functional effect of the variant alleles [14]. A NPHP3 variant predicted to influence splicing may have variable effects, depending on the transcript generated 
and any tissue-specific effects on splicing. The fact that all three affected patients we report here reached ESRD from 13-15 years of age is typical of NPHP. The variable extra renal phenotypes, including liver fibrosis, is also typical.

The original description of NPHP3 variants that effect function, leading to nephronophthisis in man, noted alternate mRNA transcripts of NPHP3 were expressed, with variation in splicing of exons $3 b, 13$, and 15 [12]. For example, the AL832877 transcript of NPHP3 is differentially spliced, omitting exon 15, and was cloned from human lymph node, whereas the clone CA396561, derived from human retinal pigment epithelium, includes exon 15 . The full-length cDNA of NPHP3 encodes for a 1330 amino acid protein. Here we have shown that in white blood cell RNA, there is alternate splicing of exon 15 of NPHP3. The consequences of loss of exon 15 , which has 83 nucleotides, is predicted to lead to a change in reading frame of the downstream exons of NPHP3 and a truncated protein of just 704 amino acids. The precise function of this predicted alternate transcript is unknown, but the transcript may be subject to nonsense-mediated decay.

This report suggests that synonymous changes in known disease causing genes (such as the known NPHP genes) cannot automatically be filtered out from WES datasets before an in silico assessment of influence on splicing has been determined. The significance of synonymous variants in $P K D 1$, one of the genes underlying autosomal dominant polycystic kidney disease, has recently been described [15]. Here two synonymous variants in PKDI were shown to induce pre-mRNA splicing defects [15]. In the case of genetically unsolved recessive cystic kidney diseases such as nephronophthisis, the finding of one variant which affects function, such as a heterozygous nonsense variant in a known genetic cause of the disease (e.g. NPHP1), should prompt a review of synonymous variants within the same gene to see if a synonymous variant may be the second allele which affects function.

\section{Conclusion}

In conclusion, we confirm the functional effect of a synonymous single-nucleotide polymorphism in NPHP3 by determining alternate splicing in kidney RNA isolated from urine-derived renal epithelial cells. This system will allow for a comprehensive comparison of kidney-specific and whole-blood RNA transcripts and for the ex vivo assessment of the functional effect of putative splice variants.

Acknowledgements This work was funded by the Northern Counties Kidney Research Fund (JAS). CAJ is supported by the Medical Research Council (MR/K011154/1). FH is supported by grants from the National Institutes of Health (DK1069274, DK1068306, and DK064614). CB receives research support for his laboratory from the Deutsche Forschungsgemeinschaft (DFG) Collaborative Research Centre (SFB) KIDGEM 1140 and the Federal Ministry of Education and Research (BMBF, 01GM1515C). We thank the patients and their families for taking part in this study.

\section{Compliance with ethical standards}

Conflict of interest The authors declare that they have no conflict of interest.

Open Access This article is licensed under a Creative Commons Attribution 4.0 International License, which permits use, sharing, adaptation, distribution and reproduction in any medium or format, as long as you give appropriate credit to the original author(s) and the source, provide a link to the Creative Commons license, and indicate if changes were made. The images or other third party material in this article are included in the article's Creative Commons license, unless indicated otherwise in a credit line to the material. If material is not included in the article's Creative Commons license and your intended use is not permitted by statutory regulation or exceeds the permitted use, you will need to obtain permission directly from the copyright holder. To view a copy of this license, visit http://creativecommons. org/licenses/by/4.0/.

\section{References}

1. Otto EA, Hurd TW, Airik R, et al. Candidate exome capture identifies mutation of SDCCAG8 as the cause of a retinal-renal ciliopathy. Nat Genet. 2010;42:840-50.

2. Chaki M, Airik R, Ghosh AK, et al. Exome capture reveals ZNF423 and CEP164 mutations, linking renal ciliopathies to DNA damage response signaling. Cell. 2012;150:533-48.

3. Gee HY, Otto EA, Hurd TW, et al. Whole-exome resequencing distinguishes cystic kidney diseases from phenocopies in renal ciliopathies. Kidney Int. 2014;85:880-7.

4. Miller TE, You L, Myerburg RJ, Benke PJ, Bishopric NH. Whole blood RNA offers a rapid, comprehensive approach to genetic diagnosis of cardiovascular diseases. Genet Med. 2007;9: 23-33.

5. Mele M, Ferreira PG, Reverter F, et al. Human genomics. The human transcriptome across tissues and individuals. Science. 2015;348:660-5.

6. Battle A, Brown CD, Engelhardt BE, Montgomery SB. Genetic effects on gene expression across human tissues. Nature. 2017; 550:204-13.

7. Daga A, Majmundar AJ, Braun DA, et al. Whole exome sequencing frequently detects a monogenic cause in early onset nephrolithiasis and nephrocalcinosis. Kidney Int. 2018;93:204-13.

8. Lu H, Galeano MCR, Ott E, et al. Mutations in DZIP1L, which encodes a ciliary-transition-zone protein, cause autosomal recessive polycystic kidney disease. Nat Genet. 2017;49:1025-34.

9. Ajzenberg H, Slaats GG, Stokman MF, et al. Non-invasive sources of cells with primary cilia from pediatric and adult patients. Cilia. 2015;4:8.

10. Srivastava S, Ramsbottom SA, Molinari E, et al. A human patientderived cellular model of Joubert syndrome reveals ciliary defects which can be rescued with targeted therapies. Hum Mol Genet. 2017;26:4657-67.

11. Fehrenbach H, Decker C, Eisenberger T, et al. Mutations in WDR19 encoding the intraflagellar transport component IFT144 
cause a broad spectrum of ciliopathies. Pediatr Nephrol. 2014;29:1451-6.

12. Olbrich H, Fliegauf $M$, Hoefele $J$, et al. Mutations in a novel gene, NPHP3, cause adolescent nephronophthisis, tapeto-retinal degeneration and hepatic fibrosis. Nat Genet. 2003;34: 455-9.

13. Tory K, Rousset-Rouviere C, Gubler MC, et al. Mutations of NPHP2 and NPHP3 in infantile nephronophthisis. Kidney Int. 2009;75:839-47.
14. Bergmann C, Fliegauf $\mathrm{M}$, Bruchle NO, et al. Loss of nephrocystin-3 function can cause embryonic lethality, MeckelGruber-like syndrome, situs inversus, and renal-hepatic-pancreatic dysplasia. Am J Hum Genet. 2008;82:959-70.

15. Claverie-Martin F, Gonzalez-Paredes FJ, Ramos-Trujillo E. Splicing defects caused by exonic mutations in PKD1 as a new mechanism of pathogenesis in autosomal dominant polycystic kidney disease. RNA Biol. 2015;12:369-74. 